\title{
Fluctuating fluid flow and heat transfer of an obliquely impinging air jet
}

\author{
Tadhg S. O'Donovan *, Darina B. Murray \\ Department of Mechanical \& Manufacturing Engineering, Trinity College Dublin, \\ Ireland
}

\begin{abstract}
Heat transfer to an obliquely impinging air jet is investigated experimentally. Distributions of the mean and the fluctuating component of the surface heat transfer are reported for a jet Reynolds number of 10000, nozzle to impingement surface distance, $H / D$, from 2 to 8 and angle of impingement, $\alpha$, from $30^{\circ}$ to $90^{\circ}$ (normal impingement). Flow velocity measurements along the impingement surface are related to heat transfer distributions. At specific locations the surface heat transfer and the local fluid velocity are measured simultaneously and coherence and phase difference information between the signals are reported. The vortical characteristic of the flow is shown to vary considerably with the angle of impingement; depending on the distance between the near nozzle edge and the impingement surface, vortices at different stages of development impact with the target surface. The influence of naturally occurring vortices in an impinging jet flow on the magnitude of heat transfer in the near wall jet is reported.
\end{abstract}

Key words: Oblique Jet Impingement, Coherence, Phase Difference, Vortices

\section{Introduction}

Obliquely impinging air jets have many applications in heat transfer due to their ability to produce high localised and area averaged heat transfer coefficients. For example, O'Donovan et al. (1) investigated the cooling of a grinding process where the research sought to determine the optimum set-up of an obliquely impinging air jet to maximise its cooling capacity. Impinging jets have also been applied to the cooling of gas turbine blades and the thermal management of electronics.

* Corresponding Author. Tel.: +353-1-896-3878; Fax.: +353-1-679-5554
Email address: tadhg.odonovan@tcd.ie (Tadhg S. O'Donovan). 
The main variables for jet impingement heat transfer are the angle of impingement, the jet Reynolds number and the height of the nozzle above the impingement surface. Comprehensive reviews of heat transfer to impinging jets have been conducted by Martin (2) and Jambunathan et al. (3) for a full range of parameters which also includes confinement, submergence, nozzle geometry and jet array configuration. Comprehensive studies of the mean fluid flow characteristics of both free and impinging jets have also been presented by Donaldson and Snedeker (4), Beltaos (5) and Martin (2).

The surface heat transfer distribution changes significantly with nozzle to impingement surface spacing. Several investigators report secondary peaks in the heat transfer distribution at low nozzle to impingement surface spacings. Goldstein and Timmers (6) compared heat transfer distributions of a large nozzle to plate spacing $(H / D=6)$ to that of a relatively small spacing $(H / D=2)$. It was shown that while the Nusselt number decays from a peak at the stagnation point for the large $H / D$, the Nusselt number is a local minimum at the stagnation point when $H / D=2$. Goldstein et al. (7) reported secondary maxima in the heat transfer distribution at nozzle to impingement surface spacings less than 5 diameters; at $H / D=2$ these maxima were greater than the stagnation point Nusselt number. The secondary maxima occur at approximately $r / D$ of 2 and were attributed to entrainment caused by vortex rings in the shear layer.

Sparrow and Lovell (8), Goldstein and Franchett (9), Yan and Saniei (10) report heat transfer distributions to an obliquely impinging air jet. At large $H / D$ the heat transfer peaks at the stagnation point, which is displaced from the geometric centre in the uphill direction (as defined in figure 1). The surface heat transfer decreases sharply in the uphill direction and more gradually in the downhill direction. In each case the asymmetry of the distributions and the displacement of the stagnation point was more pronounced with increasingly acute angles of impingement. Goldstein and Franchett (9) successfully correlated their data for a range of nozzle to impingement surface spacings in excess of 4 diameters. Yan and Saniei (10) presented results for a jet impinging at low nozzle to surface spacings and secondary peaks in the heat transfer distributions were shown to occur in the downhill direction only, for the range of parameters tested.

Gardon and Akfirat (11) successfully related the surface heat transfer to the mean velocity and turbulence intensity in a free jet at an equivalent location. Hoogendoorn (12) reported the effect of turbulence in an impinging jet flow on the resulting surface heat transfer. Data presented by Lytle and Webb (13) have shown, for $H / D \leq 1$, that a peak in velocity fluctuations parallel with the impingement surface corresponds to a peak in the mean heat transfer distribution. The most recent advances in this field, however, have been concerned with the vortex characteristics of impinging jets. In a jet flow vortices initiate 
in the shear layer due to Kelvin Helmholtz instabilities. Vortices, depending on their size and strength, affect the jet spread, the potential core length and the entrainment of ambient fluid. Since vortices also affect the magnitude and direction of velocity fluctuations close to the impingement surface, the vortical nature of an impinging jet has a significant influence on the mean heat transfer distribution.

Liu and Sullivan (14) have shown that controlling the vortices within an impinging jet flow has the potential to enhance the overall surface heat transfer. Hwang et al. (15) and Hwang and Cho (16) have used acoustic excitation to control the development of vortices in an impinging jet flow and report the influence of acoustic excitation on the surface heat transfer. O'Donovan and Murray (17), (18) have presented both mean and fluctuating heat transfer results for a normally impinging air jet. By correlating surface heat flux signals with local fluid velocity signals, the influence of naturally occurring vortices on the surface heat transfer was revealed. To date, however, the effect of vortices on surface heat transfer in an obliquely impinging jet flow has not been reported. The current investigation reports on the fluctuating heat transfer and fluid flow of an obliquely impinging air jet and sheds light on the influence naturally occurring vortices have on the mean heat transfer.

\section{Experimental Rig}

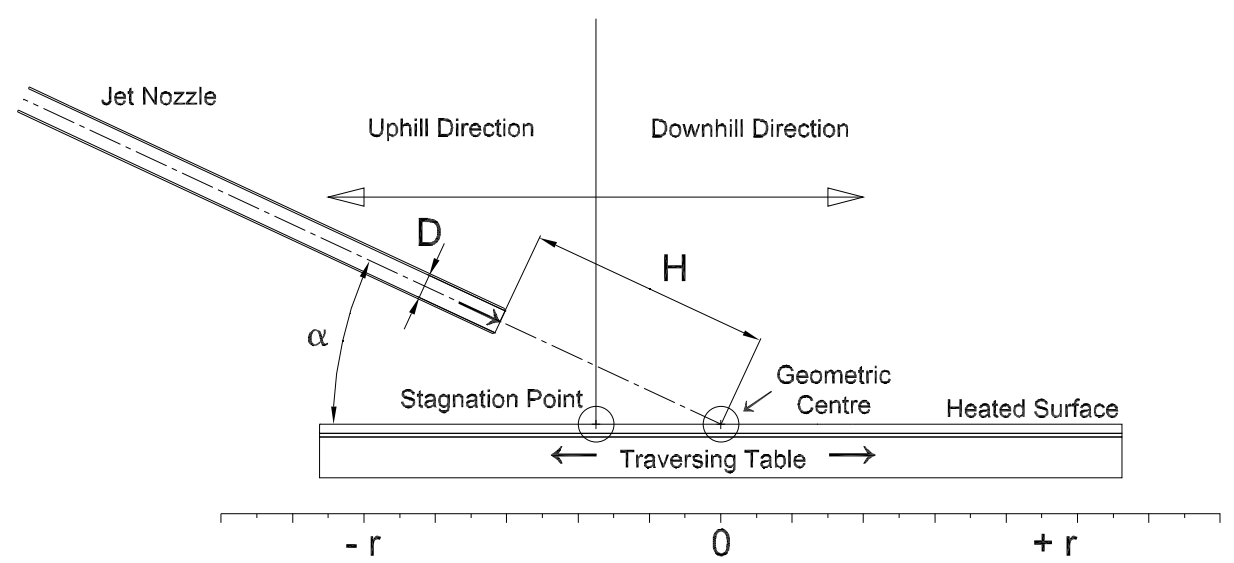

Fig. 1. Schematic of Experimental Rig

Figure 1 shows a schematic of the experimental rig which is similar to that used in an investigation by O'Donovan and Murray (17). The jet nozzle, which impinges obliquely at an angle $\alpha$, consists of a brass pipe of $13.5 \mathrm{~mm}$ internal diameter. The nozzle is clamped on a carriage in an arrangement that allows its height above the impingement surface and its angle of impingement to be varied. Air is supplied to the jet nozzle by a compressor and an Alicat Scientific Inc. Precision Gas Flow Meter is installed on the compressed air line 
to monitor both the air volume flow rate and temperature. A heat exchanger is also installed on the air line to ensure that jet exit temperature is maintained within $0.5^{\circ} \mathrm{C}$ of the ambient air temperature. The schematic illustrates two directions which are commonly referred to as the uphill and downhill directions of the flow.

A heated target surface measures $425 \mathrm{~mm} \times 550 \mathrm{~mm}$ and consists of three layers. The top surface is a $5 \mathrm{~mm}$ thick copper plate. A $15 \mathrm{~kW} / \mathrm{m}^{2}$ silicon rubber heater mat, approximately $1.1 \mathrm{~mm}$ thick, is fixed to the underside of the copper plate with a thin layer of adhesive. A thick layer of insulation prevents heat loss from the heating element other than through the copper. The thickness of the plate and the high thermal conductivity of copper ensure that the temperature of the surface remains almost constant irrespective of the variation of the heat transfer coefficient across the impingement surface. The plate approximates a uniform wall temperature boundary condition, operating typically at a surface temperature of $60^{\circ} \mathrm{C}$, however a temperature gradient of $2.5^{\circ} \mathrm{C}(6 \%$ of the temperature difference between the jet and the heated surface) has been observed over a distance of 10 diameters.

Two sensors are flush mounted on the surface to measure the surface heat flux. The first is an RdF Micro-Foil ${ }^{\circledR}$ heat flux sensor which contains a differential thermopile that measures the temperature above and below a known thermal barrier. This temperature difference is proportional to the surface heat flux. A Senflex ${ }^{\circledR}$ Hot Film Sensor is also mounted on the surface and operates in conjunction with a Constant Temperature Anemometer. This technique enables the surface heat flux to be measured at a higher temporal resolution than the Micro-Foil ${ }^{\circledR}$ sensor and can accurately acquire data in excess of $8 k H z$. This equates to a Nyquist frequency of $4096 \mathrm{~Hz}$. The equivalent Strouhal number, which is the non-dimensional form of frequency, (defined by equation 1) is calculated to be approximately 5 .

$$
S t=\frac{f D}{U_{j}}
$$

The sensor consists of a nickel sensor element that is electron beam deposited onto a $0.051 \mathrm{~mm}$ thick Upilex S polyimide film. The hot film element has a thickness of $\leq 0.2 \mu \mathrm{m}$ and covers an area of approximately $0.1 \mathrm{~mm} \times 1.4 \mathrm{~mm}$. A TSI Model 1053B Constant Temperature Anemometer is used to control the temperature of the hot film. It maintains the temperature of the film at a slight overheat $\left(\approx 5^{\circ} \mathrm{C}\right)$ above the heated surface. The power required to maintain this temperature is equal to the heat dissipated from the film. Corrections due to the overheat of the sensor above the impingement surface were made to acquire accurate measurements. Both the heated plate and the nozzle are mounted on carriages that travel on orthogonal tracks, thus allowing 
the sensor to be placed in any location in a $2 \mathrm{D}$ plane.

Two laser techniques are employed to measure the flow velocity. Particle Image Velocimetry (PIV) is employed to measure the time averaged cross-section of the velocity flow field and Laser Doppler Anemometry (LDA) is used to measure the velocity at specific locations within the impinging jet flow with greater temporal and spatial resolution. The PIV system uses a $15 \mathrm{~mJ}$ New Wave Solo Double Pulse Laser to illuminate the flow and images are captured with a double shutter PCO Sensicam camera that has a resolution of $1280 \times 1024$ pixels. The LDA system is based on a Reliant $500 \mathrm{~mW}$ Continuous Wave laser from Laser Physics. This two component system uses 2 pairs of beams, that have wavelengths of $514.5 \mathrm{~nm}$ (green) and $488 \mathrm{~nm}$ (blue), to measure the velocity in orthogonal directions at the same point location. The four beams, each of diameter $1.35 \mathrm{~mm}$, are focused on a point $250 \mathrm{~mm}$ from the laser head. The system works in backscatter mode and a Burst Spectrum Analyser (BSA) acquires and processes the signal to compute the velocity. As the LDA technique is reliant on seeding particles passing through a measurement volume the velocity signal is acquired with an irregular time step. Data presented have a minimum acquisition frequency of $8 \mathrm{kHz}$ and the raw signal has been re-sampled using Sample and Hold and a correction for error is performed according to Fitzpatrick and Simon (19).

Heat transfer results are presented in the form of the mean and root-meansquare Nusselt number which have calculated uncertainties of $\pm 5.7 \%$ and $\pm 30.1 \%$ respectively. The jet exit velocity can be set with an accuracy of $4.7 \%$. A complete calibration and uncertainty analysis for this experimental set-up is presented by O'Donovan (20).

\section{Results \& Discussion}

Time averaged fluid flow data of an obliquely impinging air jet are used to explain the resulting surface heat transfer. Certain locations within the wall jet of the impingement flow warranted further investigation and therefore a temporal analysis of the surface heat flux and local velocity signal was conducted. These data are presented in the following two sections.

\subsection{Time-Averaged Fluid Flow \& Heat Transfer Measurements}

The flow field of interest in this investigation is a cross-section through the centreline of the impinging jet flow. Figure 2 presents velocity flow field measurements for a jet impinging at $H / D=6$ and a range of oblique angles. A 

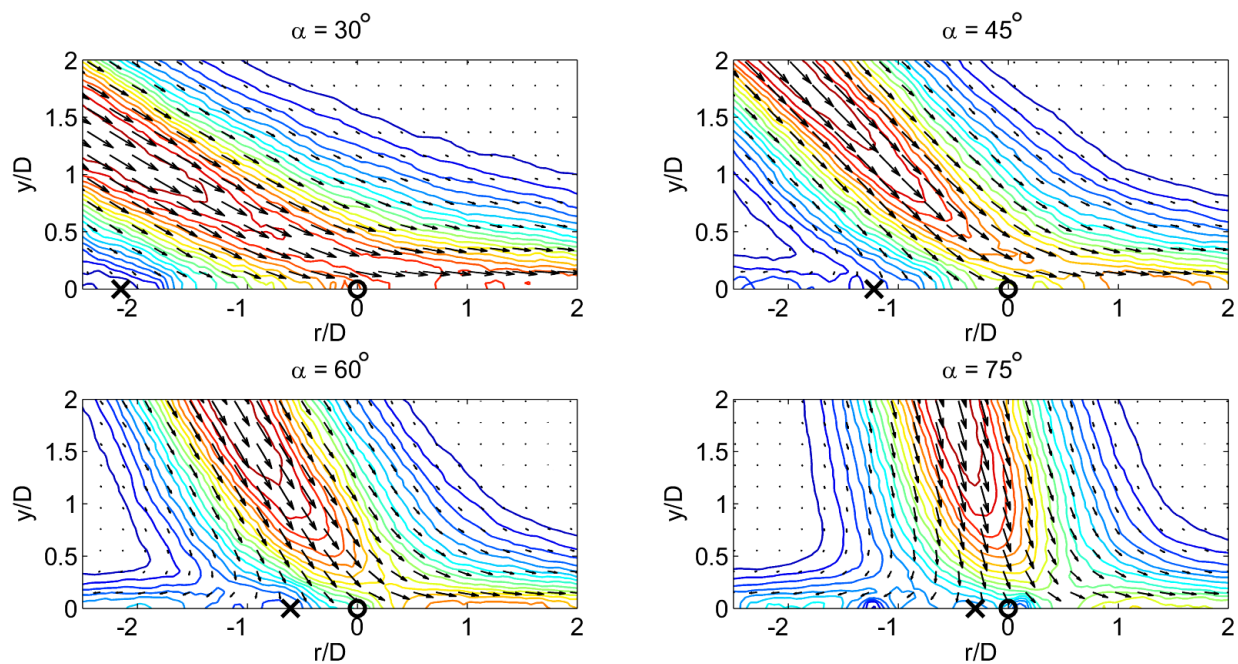

Fig. 2. Velocity Flow Fields for an Obliquely Impinging Jet, $H / D=6$

circle and an $\mathrm{x}$ indicate the location of the geometric centre and the stagnation point respectively. As the angle made with the impingement surface becomes more acute the stagnation point moves further from the geometric centre in the uphill direction (as defined in figure 1).

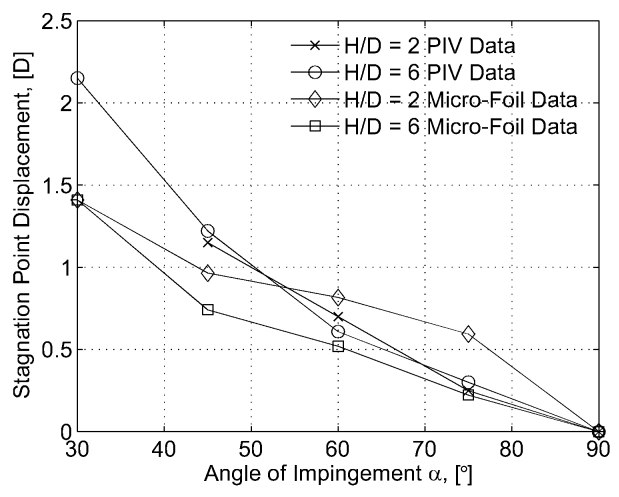

(a) Experimental Data

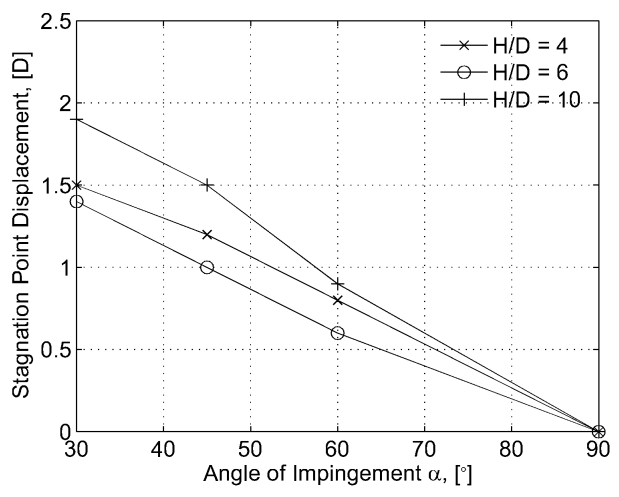

(b) Goldstein \& Franchett (9)

Fig. 3. Displacement of Stagnation Point from Geometric Centre

The displacement of the stagnation point from the geometric centre has also been observed for $H / D=2$ and these data are plotted in figure 3 (a), which also indicates the location of the maximum heat transfer for the same range of parameters. These data compare favourably to the findings of Goldstein and Franchett (9) who observed that the peak in the surface heat transfer distribution was displaced from the geometric centre and associated it with the location of the stagnation point. It is clear that the stagnation point location is dependent on the angle of impingement; however the height of the jet above the plate appears to have negligible influence as evidenced by the PIV data. The location of the peak heat transfer follows a similar trend but has a greater 
spread; this has been attributed to the positioning accuracy of the Micro-Foil ${ }^{\circledR}$ sensor. Although the variation of the stagnation point displacement is not discussed by Goldstein and Franchett (9) in detail, an empirical equation that correlates their experimental data, for a wider range of $H / D$ than the current investigation, shows a slight dependence on the nozzle to impingement surface spacing. This variation of stagnation point displacement from the geometric centre is illustrated in figure 3 (b). In an investigation reported by Beitelmal et al. (21), the displacement of the stagnation point was found to be independent of Reynolds number.

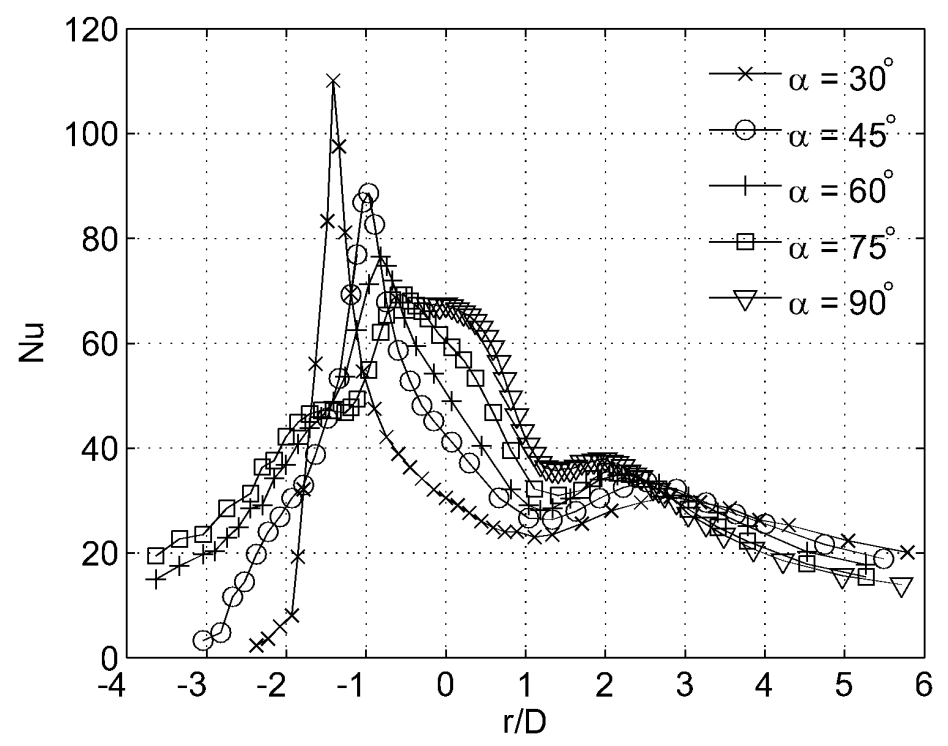

(a) $H / D=2$

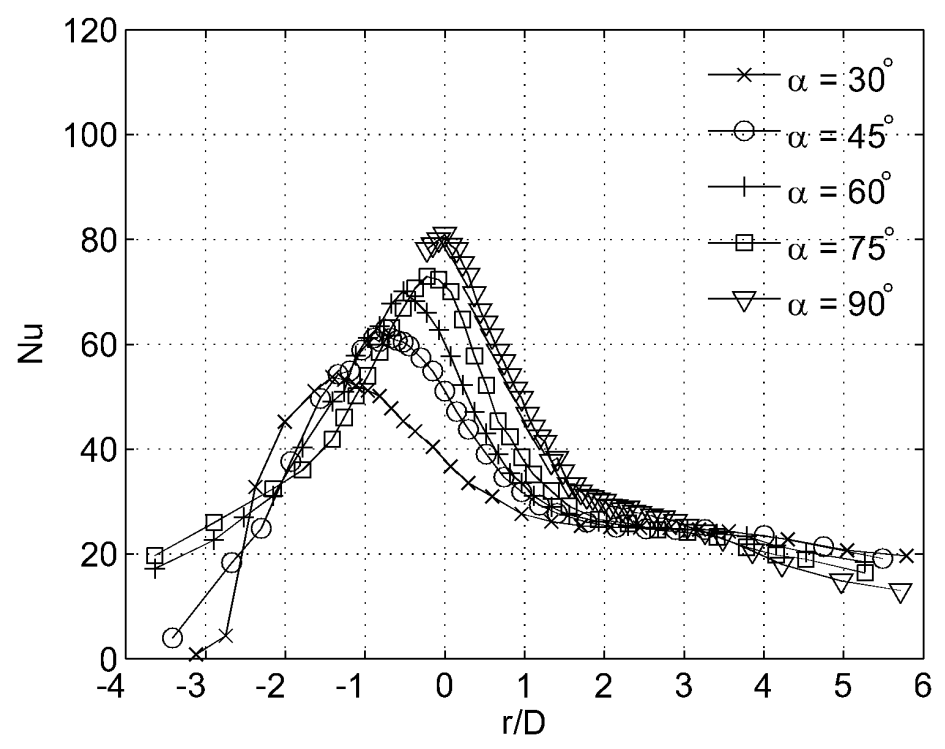

(b) $H / D=6$

Fig. 4. Mean Nusselt Number Distributions for Oblique Angles of Impingement 


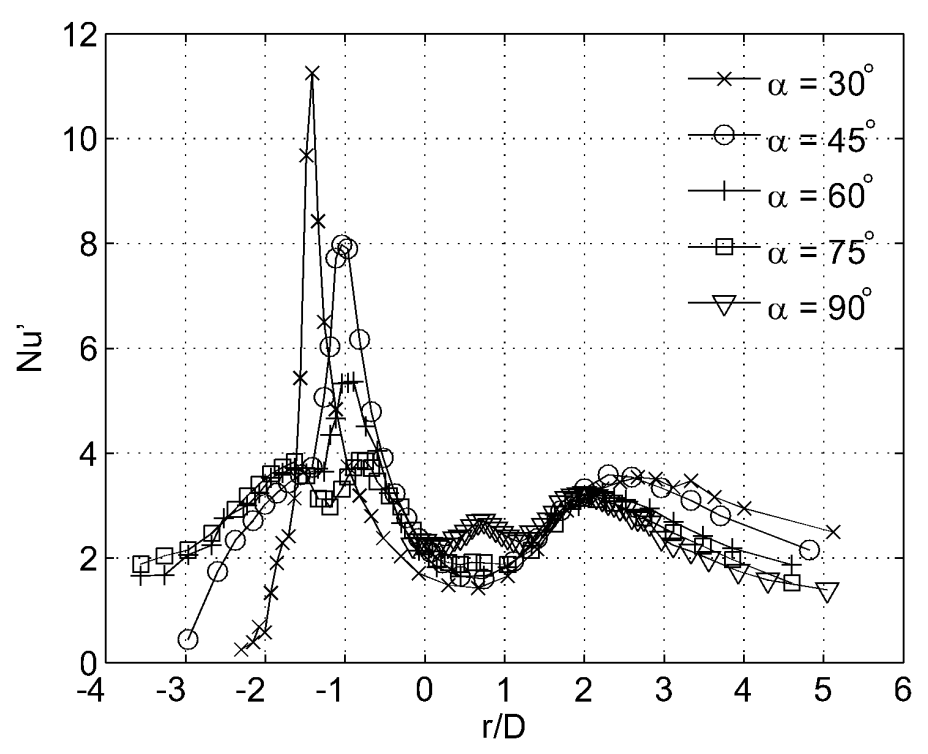

(a) $H / D=2$

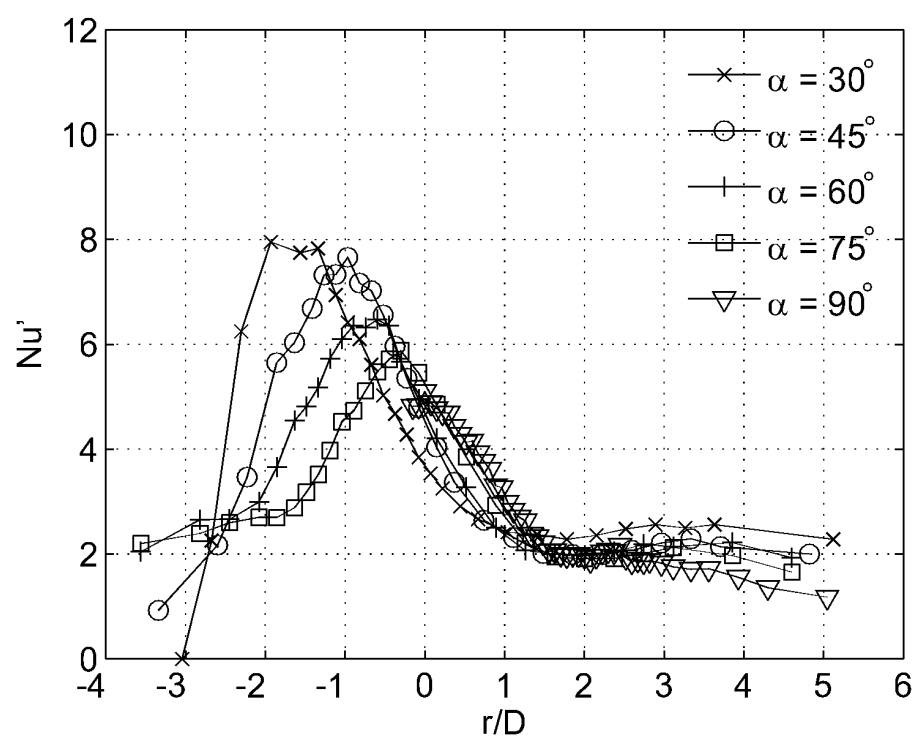

(b) $H / D=6$

Fig. 5. RMS Nusselt Number Distributions for Oblique Angles of Impingement

Heat transfer distributions for a jet impinging at a range of oblique angles are presented in figure 4 (a) and (b) for normalised jet to surface spacings of $H / D=2$ and $H / D=6$ respectively. The first spacing corresponds to a location where the impingement surface is within the potential core of the jet, and the second spacing to a location just beyond the core of the jet. It is apparent from the heat transfer distributions that the peak heat transfer occurs at the stagnation point as it is displaced an equivalent distance in the uphill direction from the geometric centre. One exception to this occurs at an angle of impingement, $\alpha=30^{\circ}$. It can be seen from the velocity flow fields 
presented in figure 2 that the stagnation point occurs at approximately $r / D=$ -2.1 . However, the peak in both the $N u$ and $N u^{\prime}$ distributions (figures 4, 5 ) occurs at approximately $r / D=-1.4$ for $\alpha=30^{\circ}$. This discrepancy is explained by considering the velocities along the impingement surface at $\alpha=$ $30^{\circ}$. At this acute angle of impingement the local velocity close to the surface decreases from $r / D=0$ to the point where it is almost negligible at $r / D=$ -1.4 even though true stagnation does not occur until $r / D=-2.1$. Therefore the peak heat transfer occurs at $r / D=-1.4$ where fluctuations in the flow, or the instantaneous velocity, remains high. Although the spatial resolution of the measurements presented in the current investigation does not allow, it is anticipated that this effect would be present, to a lesser extent for the larger angles of impingement. The results also show that, for $H / D=2$, the smaller angles of impingement produce the highest stagnation point heat transfer. The opposite is true at $H / D=6$ where the maximum heat transfer occurs when the jet impinges normally to the surface.

It is well documented by Goldstein et al. (7), Baughn and Shimizu (22), Huang and El-Genk (23) and others that at low nozzle to impingement surface spacings, secondary peaks in the Nusselt number distribution occur at a radial location of approximately 1 to 3 diameters from the geometric centre for a normally impinging jet. Figure 4 (a) shows that these peaks in the Nusselt number distribution occur for oblique jet impingement also but this feature is not present in the data shown in Figure 4 (b) where $H / D=6$. It can be seen also from the results presented in figure 4 (a) that secondary peaks may not occur in the uphill direction. For example, when the jet impinges at an oblique angle of $30^{\circ}$ there is no secondary peak in the uphill direction but a broad secondary peak does occur in the downhill direction. As the angle of impingement increases towards normal impingement a narrow secondary peak appears in the uphill direction. The magnitude of this peak is greater than that in the downhill direction.

Secondary peaks have been attributed to increased turbulence in the wall jet boundary layer and therefore the turbulence characteristic of the flow in both uphill and downhill directions is of interest. The root-mean-square Nusselt number distributions reflect the level of local fluid temperature and velocity fluctuations (turbulence) near the heated surface. These fluctuating Nusselt numbers are presented in figure 5 for the same range of parameters as in figure 4. The rms profiles are broadly similar to the mean heat transfer profiles, indicating that the Nusselt number is influenced by the level of fluctuations in heat transfer and hence by the turbulence intensity close to the impingement surface. This is consistent with expectations. Also, it can be seen that the secondary peaks in the mean Nusselt number distributions at $H / D=2$ correspond to sharp gradients in the corresponding $N u^{\prime}$ distributions, suggesting abrupt increases in wall jet turbulence at these locations. Two radial peaks occur in the $N u^{\prime}$ distribution for the normally impinging jet $\left(\alpha=90^{\circ}\right)$. 
This is explained by O'Donovan and Murray (17) to be due to the shear layer interaction with the wall jet.

At $H / D=2$, the greatest $N u^{\prime}$ magnitudes correspond with the greatest mean $N u$ magnitudes; this is not the case for $H / D=6$. While the radial positions of the peaks in the mean and fluctuating heat transfer distributions match for the latter case, the fluctuations are greatest for the most acute angle $\left(30^{\circ}\right)$ whereas the opposite is true for the mean Nusselt number. The conclusion that can be drawn from this analysis is that the controlling influence on heat transfer in this case is not the magnitude of velocity and temperature fluctuations but, perhaps, the mean flow velocities. Evidence is presented in section 3.2 that structures within the impinging jet flow also have an influence on the surface heat transfer.

The effect of $H / D$ on the heat transfer distribution for a single angle of impingement $\left(45^{\circ}\right)$ and Reynolds number $(10000)$ is presented in figure 6. Increasing the nozzle to impingement surface spacing has the effect of decreasing the magnitude of the peak Nusselt number that occurs at the stagnation point. As expected the increased spacing also diminishes the magnitude of the secondary peak, until there is no discernable change in slope in the downhill direction at $H / D=8$. Figure 6 (b) presents the root-mean-square Nusselt number distributions for the same range of parameters. By comparing the rms and mean heat transfer distributions it appears that peaks and troughs in both profiles occur at the same location. The peaks in the rms heat transfer are more pronounced, however, indicating that the level of heat transfer fluctuation is not the only factor influencing the mean heat transfer. The same inference can be drawn from the fact that $N u^{\prime}$ at the stagnation point is almost independent of jet to plate spacing whereas the peak in the mean Nusselt number distribution varies considerably with $H / D$.

\subsection{Temporal Analysis of Heat Transfer and Local Fluid Velocity}

In order to understand the dominant convective heat transfer mechanisms at play, the air velocity was measured at a distance of approximately $3 \mathrm{~mm}$ from the impingement surface, using a 2 component laser Doppler anemometer (LDA). Figure 7 presents mean and root-mean-square axial and radial velocity measurements together with the corresponding Nusselt number distributions for a jet Reynolds number of 10000 impinging at an oblique angle of $45^{\circ}$ and $H / D=2$. A schematic of the test setup is provided in figure 8 .

It can be seen from figure 7 (a) that the mean axial velocity is a maximum at $r / D \approx-0.5$ which is on the downhill side of the stagnation point; the axial velocity decreases sharply in the uphill direction and more gradually in 


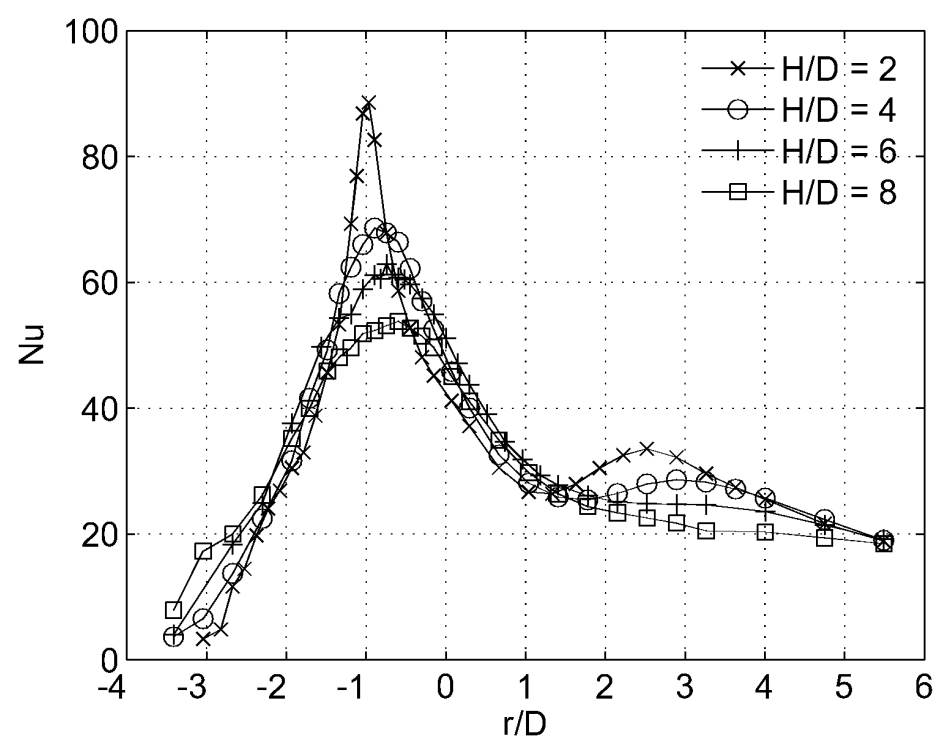

(a) Mean $N u$

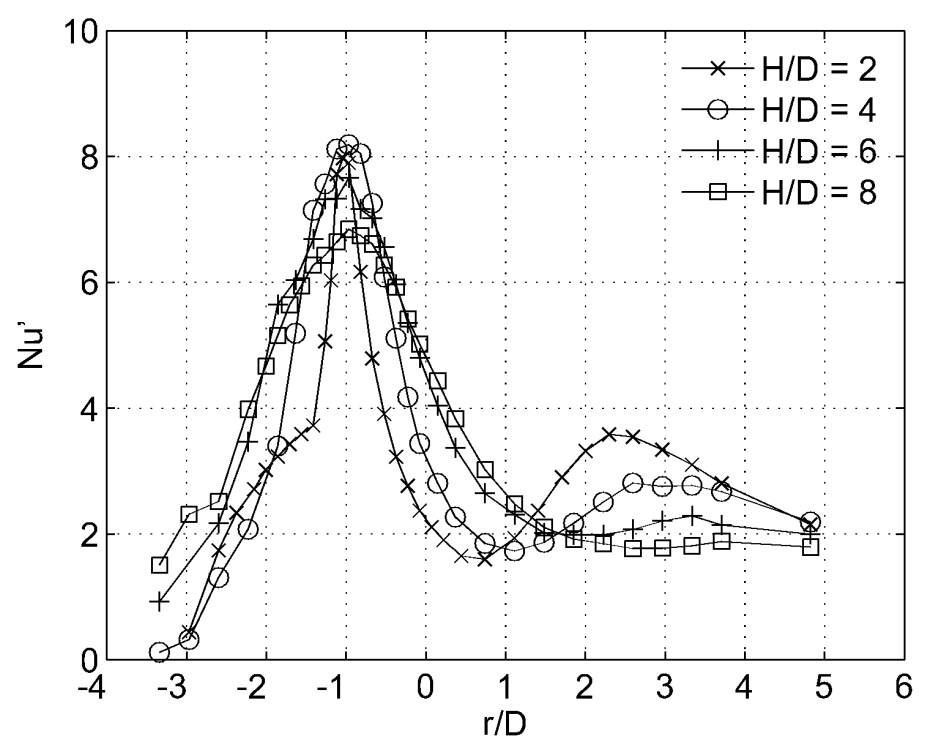

(b) Root-Mean-Square $N u$

Fig. 6. Nusselt Number Distributions for Oblique Impingement; $R e=10000$; $\alpha=45^{\circ}$

the downhill direction. The radial velocity is zero at the stagnation point, as expected, and increases with increasing radial location. The negative velocities presented simply indicate the flow direction is from right to left. The magnitude of the velocity is greater in the downhill direction as this is the direction of the main flow. The radial velocity increases to a peak at $r / D=1$ and then decreases as the jet flow spreads radially. The magnitudes of the axial and radial rms velocities are similar throughout the distribution. The velocity fluctuations are high, relative to their mean, at the stagnation point 


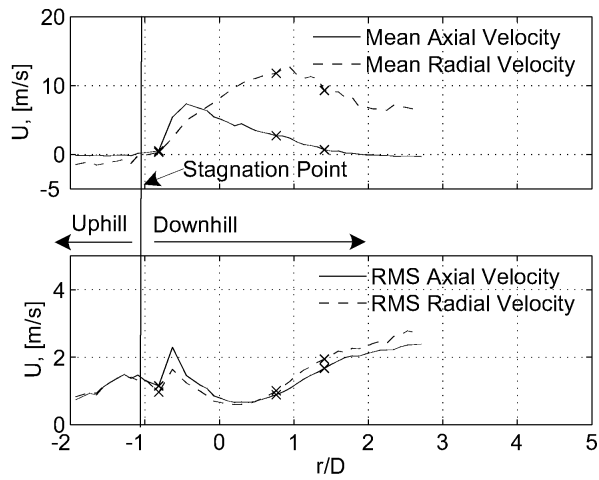

(a) Velocity

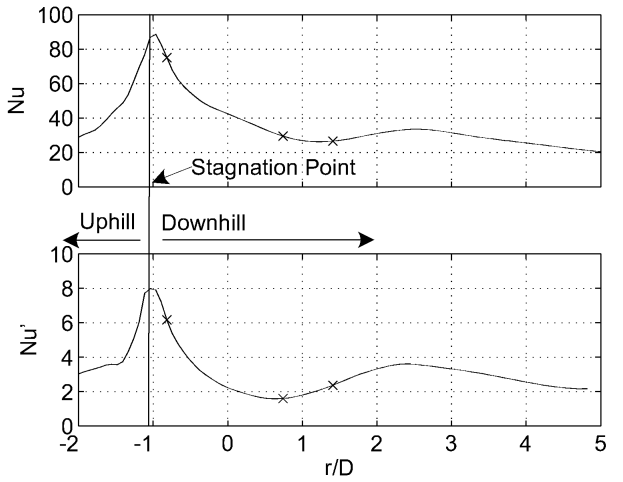

(b) Nusselt Number

Fig. 7. Velocity \& Heat Transfer Distributions; $H / D=2 ; \alpha=45^{\circ}$

which contributes to a peak in the mean Nusselt number distribution (figure 7 (b)) at this location. A combination of high mean radial velocity and high root-mean-square velocities results in a secondary peak in the heat transfer distribution at $r / D=2.25$.

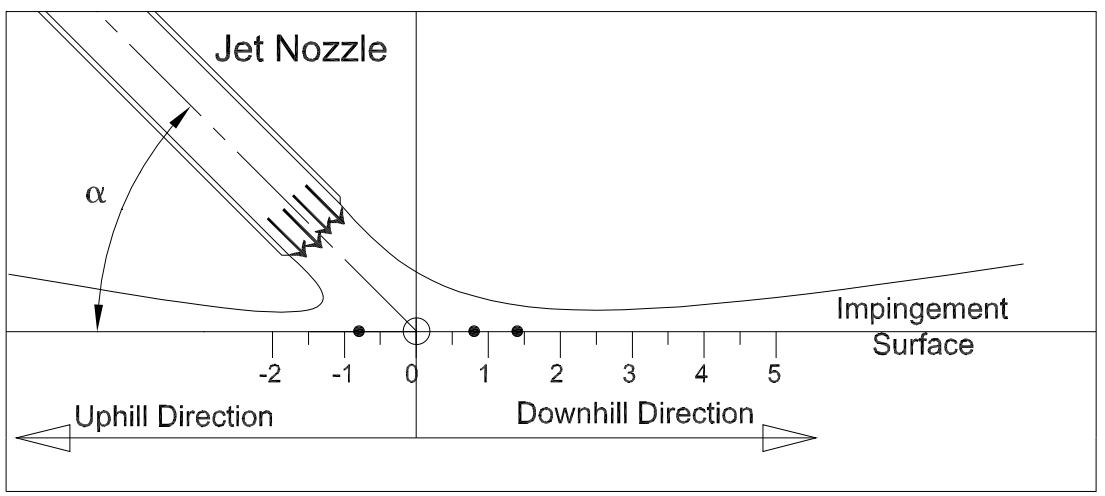

Fig. 8. Schematic of Test Setup \& Impinging Jet Flow; $H / D=2 ; \alpha=45^{\circ}$

In order to understand the relationship between convective heat transfer and fluid velocity better, temporally simultaneous measurements of surface heat flux and local fluid velocities were made at various radial locations. In the case where the jet impinges at an angle of $45^{\circ}$, the measurements were acquired at $r / D=-0.8,0.8$ and 1.4 (all on the downhill side). These locations are marked on the heat transfer and velocity distributions in figure 7 . This focus on the downhill direction is primarily due to the low magnitude of the velocity in the uphill direction for the oblique angle of impingement of $45^{\circ}$. Spectral analysis was carried out on both velocity signals and on the heat flux signal. The velocity and heat flux signals were also cross-correlated to calculate the coherence and phase difference between the signals. Results for each radial location are presented in figures 9 to 11 . 

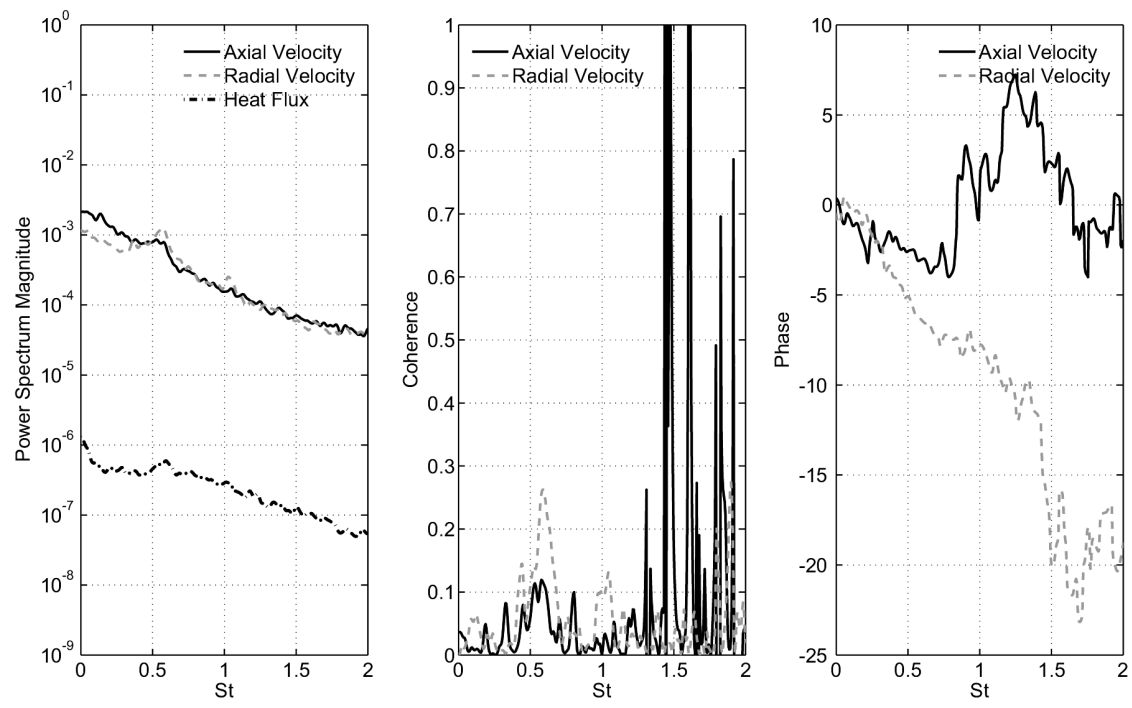

Fig. 9. Power spectra and coherence and phase information between velocity and heat flux signals; $H / D=2, \alpha=45^{\circ}, r / D=-0.8$
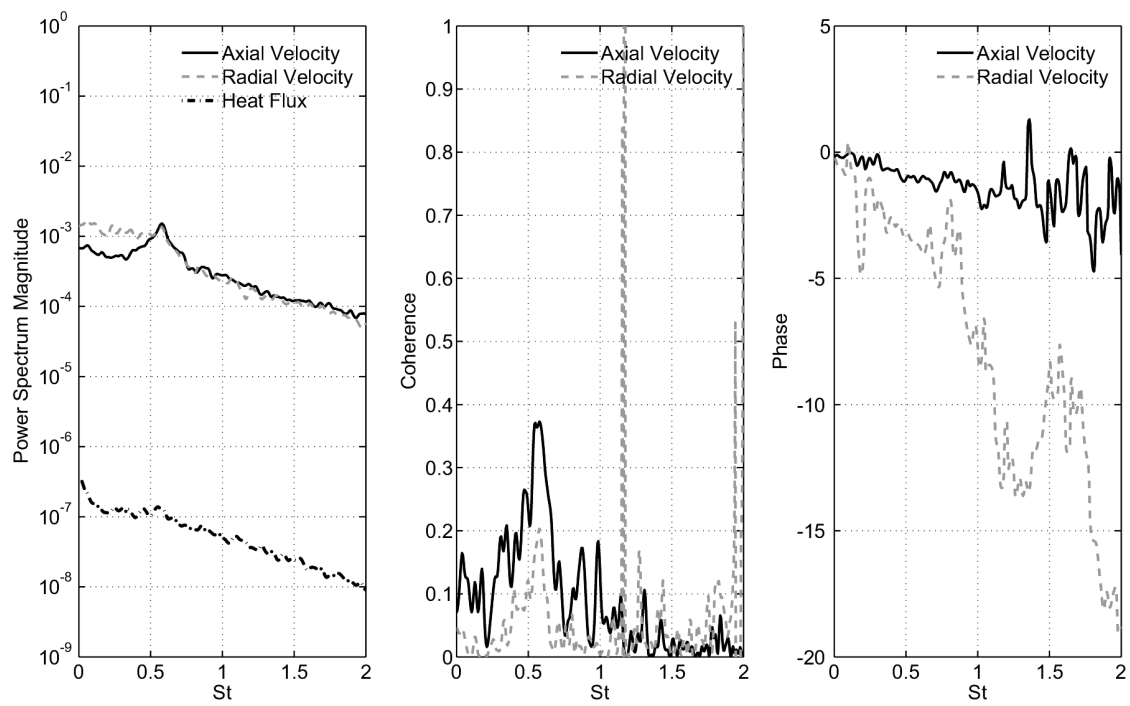

Fig. 10. Power spectra and coherence and phase information between velocity and heat flux signals; $H / D=2, \alpha=45^{\circ}, r / D=0.8$

While peaks in the heat flux and velocity spectra presented in figure 9 for $r / D=-0.8$ are small, the coherence between the signals exhibits significant peaks at Strouhal numbers of 0.6 and 1.1, indicating the passing frequency of vortices at this point. At Strouhal numbers greater than 1.3 a series of single point peaks in coherence occur, however the high coherence at these points are dismissed as an artifact of the data processing. Throughout the results presented, single point coherence approaching unity are also dismissed. 

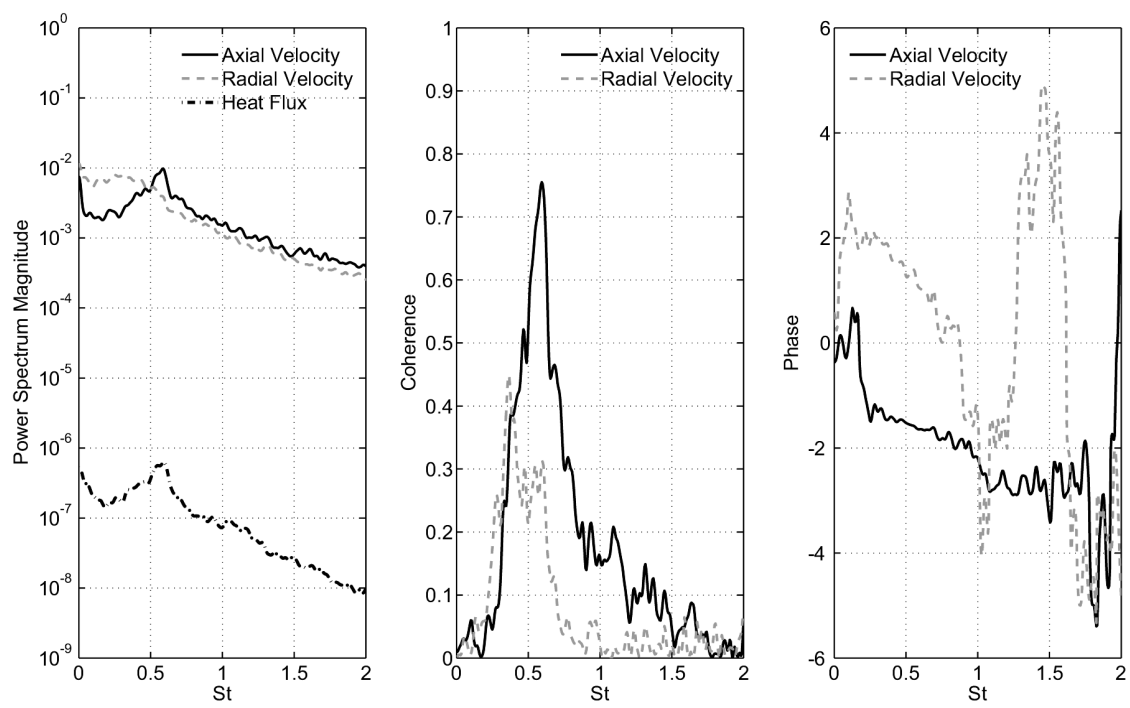

Fig. 11. Power spectra and coherence and phase information between velocity and heat flux signals; $H / D=2, \alpha=45^{\circ}, r / D=1.4$

O'Donovan and Murray (18) have shown that vortices initiate at the jet exit at a Strouhal number of 1.6 and undergo merging processes as they travel downstream. Merging has the effect of increasing the size of the vortices and decreasing their strength and passing frequency. At $r / D=-0.8$ the local flow velocity and heat flux signals are not influenced greatly by vortices. The schematic presented in figure 8 shows that vortices passing in the uphill direction will not impinge at this radial location. The peaks that occur in the signal spectra at this location have propagated through the main flow, are weak and therefore have little influence on the heat flux signal as indicated by the low overall coherence between the signals.

Further along the wall jet, in the downhill direction, vortices travel a greater distance and therefore have more time to merge before impinging on the surface, again this can easily be seen by referring to figure 8 . This is evident from data presented in figures 10 and 11 for $r / D=0.8$ and 1.4 respectively. One dominant frequency peak appears in the spectra at a Strouhal number of 0.6 and therefore it is clear that the higher frequency vortices have merged. At $r / D=1.4$ the coherence between the axial velocity and the heat flux is particularly high, indicating the influence that velocity fluctuations normal to the surface have on the heat flux. This highlights the effect that relatively small fluctuations in the axial direction have on the heat flux. Generally, it has been found that the coherence between the axial velocity and the heat flux signal is greater than that between the radial velocity and the heat flux. This is especially noticeable in figure 11, which corresponds to a location 1.4 diameters from the geometric centre, where the radial component has a greater mean velocity and similar rms velocity to the axial component. The greater coher- 
ence is attributed to a stronger thermal gradient normal to the impingement surface (axial direction) rather than parallel to the surface (radial direction).

The phase difference is calculated between the individual velocity signals and the surface heat flux, however, the coherence high over a sufficient Strouhal number range for the phase to be accurate. The slope of the phase then enables the convection velocity to be calculated using equation 2 :

$$
U_{c}=\frac{2 \pi \delta f}{\phi}
$$

where $\delta$ is the distance between the velocity and surface heat flux measurement points, $f$ is the frequency at which the convection velocity is calculated and $\phi$ is the phase difference between the two signals. As the slope of the phase increases the convection velocity decreases, therefore data presented in figures 10 and 11 show that fluctuations in the axial direction convect towards the heated surface more quickly than fluctuations in the radial direction. This also supports the assertion that fluctuations normal to the impingement surface have a greater influence on the surface heat transfer.

A similar analysis has been carried out for a jet impinging at an angle of $60^{\circ}$. The distribution of the velocities and the heat transfer profiles are presented in figure 12. Spectral and coherence analysis has been carried out at $r / D=-1.3$, -1.1 (both uphill side) and at $r / D=0.4,1.1$ (both downhill side) and results are presented in figures 13 to 16. The phase information presented here for a jet impinging at an angle of $60^{\circ}$ is consistent with that for $\alpha=45^{\circ}$ and also indicates the dominance of velocity fluctuations normal to the surface over parallel velocity fluctuations.

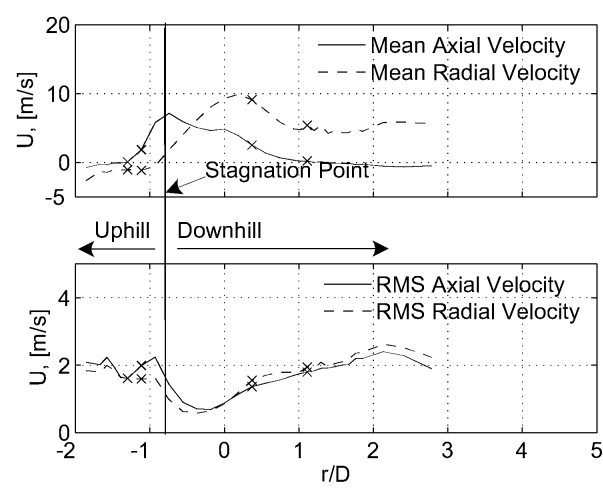

(a) Velocity

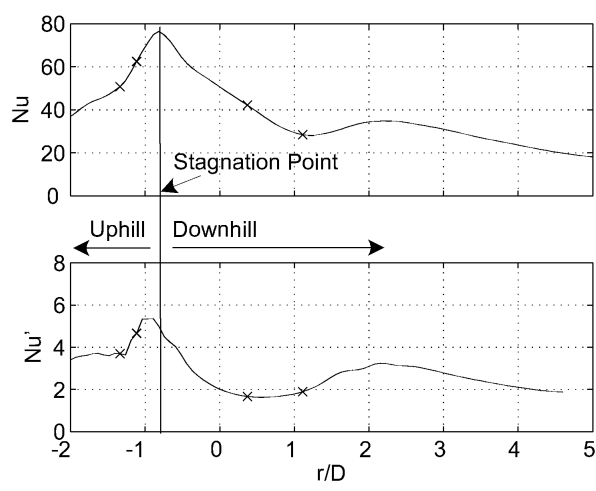

(b) Nusselt Number

Fig. 12. Velocity \& Heat Transfer Distributions; $H / D=2 ; \alpha=60^{\circ}$

It can be seen from figure 12 (a) that the magnitudes of the flow velocities in the uphill direction are small relative to those in the downhill direction. The heat transfer and velocity fluctuations in this region remain relatively high, 

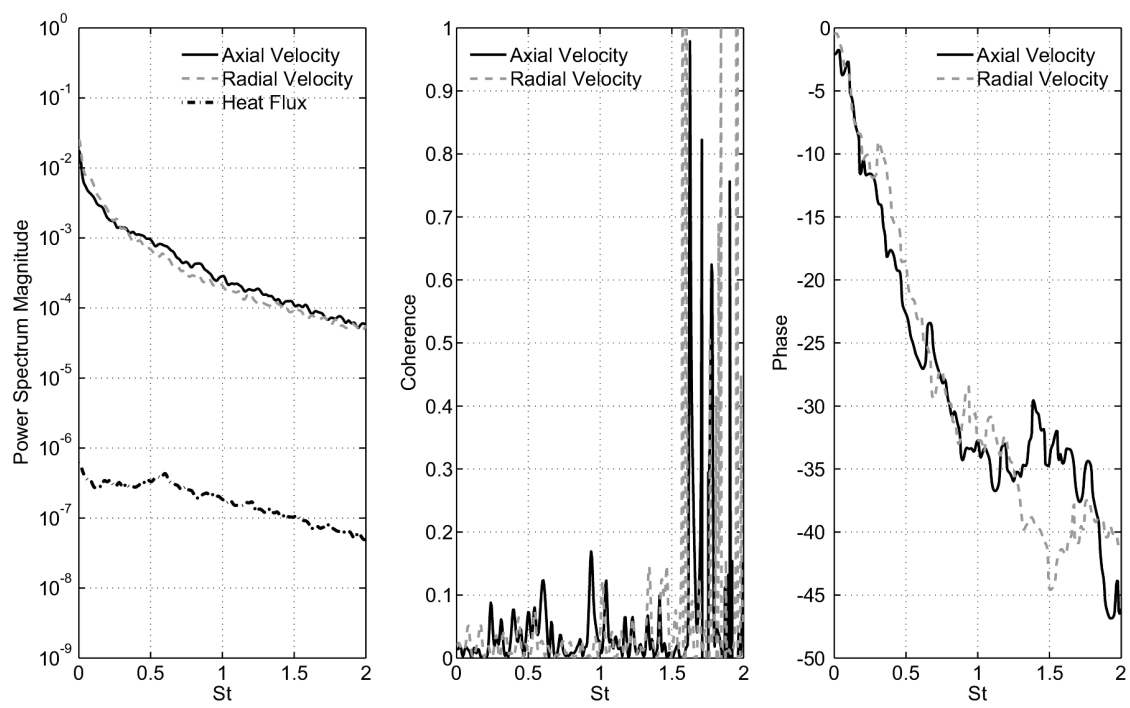

Fig. 13. Power spectra and coherence and phase information between velocity and heat flux signals; $H / D=2, \alpha=60^{\circ}, r / D=-1.3$

however. The spectral, coherence and phase information exhibit many of the characteristics discussed earlier for a jet impinging at $45^{\circ}$. While peaks in the heat transfer and velocity spectra in the uphill region are very small, it can be seen from the coherence between the velocity and heat flux signals in figure 14 that vortices pass in this direction at a Strouhal number of approximately 1.6. Figures 13 and 14 also exhibit smaller peaks at $S t=1$ and 0.5 indicating that some merging of vortices has occurred. O'Donovan and Murray (18) have shown that vortices in an impinging jet flow break-up shortly after impact with the surface. The vortex break-up location indicates a transition to turbulent flow that cannot sustain large scale flow structures. According to O'Donovan and Murray (18) the break-up of strong vortices which have not undergone a series of merging processes leads to an increase in magnitude of the secondary peak. As the heat transfer in the uphill direction decreases rapidly, due to the low mean velocities, this enhancement of heat transfer is thought to manifest itself as a slight change in slope at a radial location of approximately $r / D=$ -1.5 (figure 12 (b)). Essentially, the break-up of the strong vortex structure in this region is responsible for the relatively large magnitude of turbulence and fluctuating heat transfer in this region.

Figures 15 and 16 present equivalent data for the downhill direction of the wall jet flow. It is apparent that the vortices that pass in this direction are larger as they have a greater influence on the surface heat flux and velocity signals. At a radial location of $0.4 D$ (figure 15) from the geometric centre, peaks in all three spectra are evident at a Strouhal number of 0.6. Therefore the vortices, which have traveled a greater distance in this case before impacting with the surface, have most likely undergone a series of merging processes to reduce 

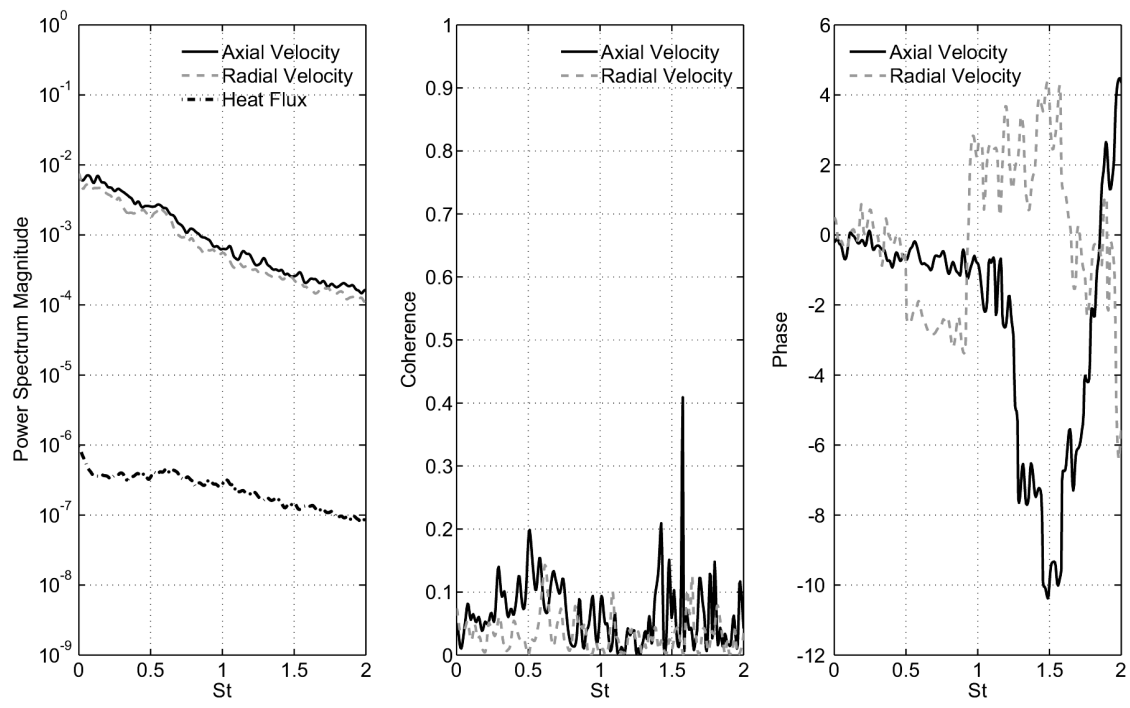

Fig. 14. Power spectra and coherence and phase information between velocity and heat flux signals; $H / D=2, \alpha=60^{\circ}, r / D=-1.1$
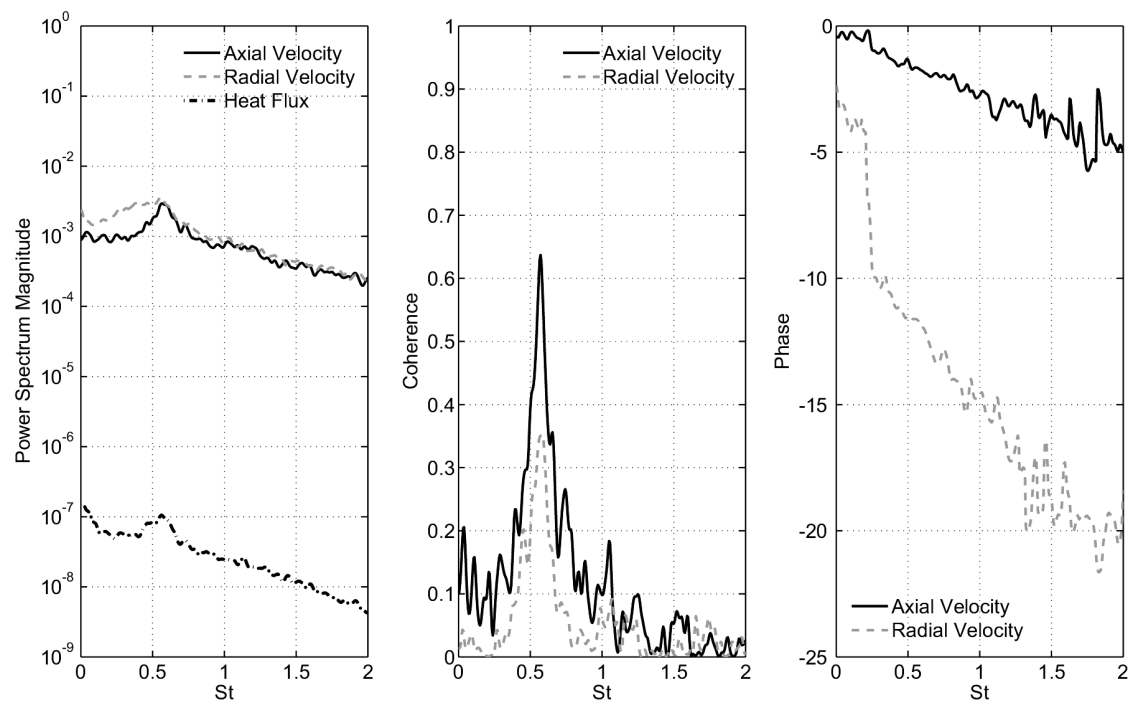

Fig. 15. Power spectra and coherence and phase information between velocity and heat flux signals; $H / D=2, \alpha=60^{\circ}, r / D=0.4$

their passing Strouhal number. Similar findings are presented in figure 16 for a location $r / D=1.1$ In this case the peak in the coherence between the axial velocity and the heat flux is broader and is still centred at a Strouhal number of 0.6. The peak in the coherence between the radial velocity and the heat flux however is shifted to approximately 0.4 indicating that the vortices are undergoing yet another merging process before being broken down into random small scale turbulence. This is consistent with the findings of Orlandi 

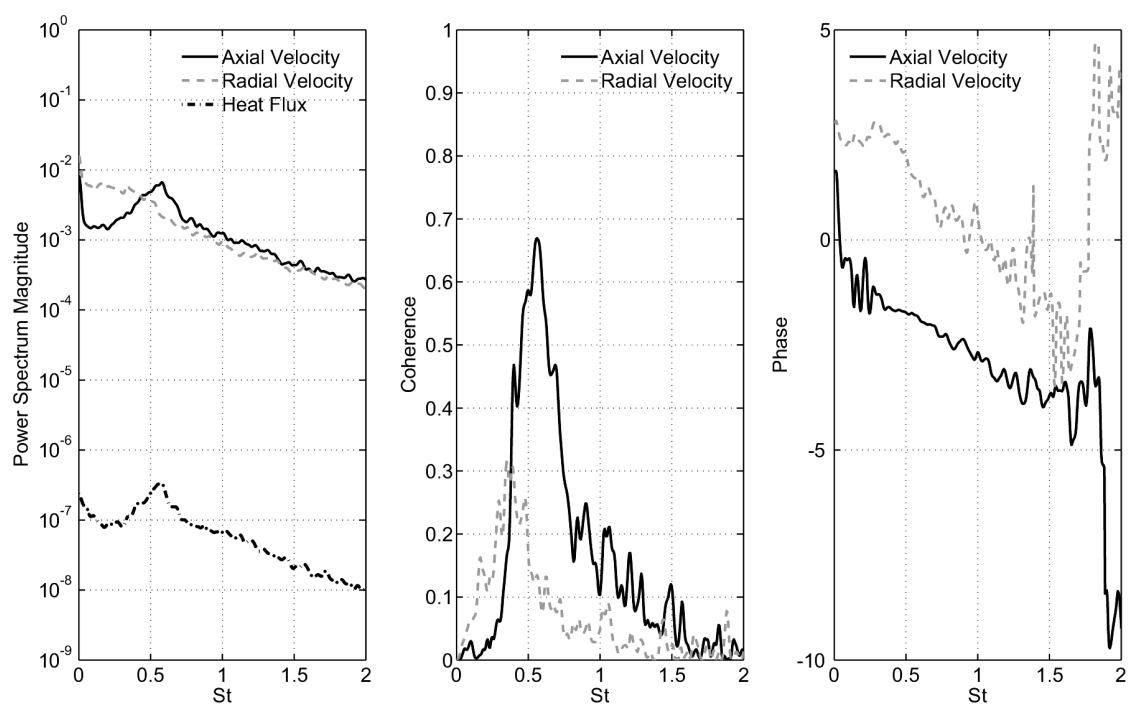

Fig. 16. Power spectra and coherence and phase information between velocity and heat flux signals; $H / D=2, \alpha=60^{\circ}, r / D=1.1$

and Verzicco (24) who have shown that merging vortices present as one large vortex in the radial direction, while remaining separate entities in the axial direction.

Since these data show that the vortices in this direction are large and pass at a low frequency, the hypothesis of O'Donovan and Murray (18) suggest that as they break-up in the wall jet they should not instigate as high a degree of turbulent fluctuations normal to the surface. These results support that hypothesis as the enhancement of the heat transfer to a secondary peak is quite modest in the downhill direction and takes the form of a broad subtle secondary peak. A comparison can be made between this peak in the downhill direction at $r / D=2.2$ and the equivalent location in the uphill direction at $r / D=-1.5$. At both locations the mean axial velocities are similar. The radial velocity in the downhill direction is much larger but this does not result in a higher local Nusselt number. Therefore it is suggested that the break-up of vortices in the wall jet, in the uphill direction where the vortices have not yet fully merged, maintains the heat transfer higher at the uphill location than at the downhill location.

\section{Conclusions}

Mean and rms Nusselt number distributions have been presented for a jet Reynolds number of 10000 , non-dimensional surface to jet exit spacings from 2 to 8 and oblique angles of impingement from $30^{\circ}$ to $90^{\circ}$ (normal impinge- 
ment). Particle image velocimetry has been employed to measure the fluid flow in a two-dimensional plane through a section of the impinging jet flow. Comparison of the flow data to heat transfer distributions has shown that the maximum heat transfer occurs at the stagnation point, which is displaced in the uphill direction from the geometric centre for oblique angles of impingement. The magnitude of the displacement is inversely proportional to the angle of impingement and is not influenced by the height of the nozzle above the impingement surface.

The root-mean-square Nusselt number is a measure of the surface heat transfer fluctuations which reflect the level of local fluid temperature and velocity fluctuations (turbulence) near the heated surface. These distributions have shed light on some of the convective heat transfer mechanisms which occur with an impinging air jet. While some of the $N u^{\prime}$ trends correspond to the $N u$ distributions, it is clear that the magnitude of the fluctuations is only one factor in determining the overall mean heat transfer. Measurements of the fluid velocity in the wall jet close to the impingement surface have also been presented and used to explain some of the trends in the mean heat transfer distributions.

An investigation of the temporal nature of the heat transfer and fluid was conducted to measure the extent to which flow structures within the impinging jet flow influenced the mean heat transfer distribution. Analysis of simultaneous heat transfer and velocity measurements has shown that velocity fluctuations normal to the impingement surface have a more significant influence on the heat transfer. Spectral analysis of the heat transfer and velocity signals has shown the effect of vortices on the heat transfer. At oblique angles of impingement the vortical characteristic of the flow and consequently the local heat transfer is different in the uphill and downhill directions. This is due to the proximity of the jet nozzle to the impingement surface. In the downhill direction the vortices have time and space to develop and pass as large scale low frequency vortices. In the uphill direction however, the vortices have not completed a merging process and pass as small scale high frequency vortices. The break-up of vortices in the wall jet influences convective heat transfer in this region. 


\begin{tabular}{|c|c|c|}
\hline Symbol & Description & Units \\
\hline$D$ & diameter & {$[m]$} \\
\hline$f$ & frequency & {$[H z]$} \\
\hline$h$ & convective heat transfer coefficient & {$\left[W / m^{2} K\right.$} \\
\hline$H$ & height of nozzle above surface & {$[m]$} \\
\hline$k$ & thermal conductivity & {$[W / m K]$} \\
\hline$N u$ & Nusselt number, $h D / k$ & {$[-]$} \\
\hline$N u^{\prime}$ & root-mean-square Nusselt number & {$[-]$} \\
\hline$r$ & radial distance from geometric centre & {$[m]$} \\
\hline$R e$ & jet Reynolds number, $\rho U D / \mu$ & {$[-]$} \\
\hline$S t$ & Strouhal number, $f D / U$ & {$[-]$} \\
\hline$U$ & velocity & {$[\mathrm{m} / \mathrm{s}]$} \\
\hline \multicolumn{3}{|c|}{ Greek Symbols } \\
\hline$\alpha$ & angle of impingement & {$\left[{ }^{\circ}\right]$} \\
\hline$\delta$ & distance between measurement points & {$[m]$} \\
\hline$\mu$ & viscosity & {$[k g / m s]$} \\
\hline$\rho$ & density & {$\left[\mathrm{kg} / \mathrm{m}^{3}\right]$} \\
\hline$\Phi$ & Phase & {$[-]$} \\
\hline \multicolumn{3}{|c|}{ Subscripts } \\
\hline$c$ & convection & \\
\hline$j$ & jet & \\
\hline
\end{tabular}

\section{Acknowledgment}

This work was supported in part by Enterprise Ireland under grant SC/2001/0071 and also by Science Foundation Ireland grant 04/BR/EO108.

\section{References}

[1] T. S. O'Donovan, D. B. Murray, A. A. Torrance, Jet heat transfer in the vicinity of a rotating grinding wheel, Proceedings of the Institution of 
Mechanical Engineers Part C - Journal of Mechanical Engineering Science 220 (2006) $837-845$.

[2] H. Martin, Heat and mass transfer between impinging gas jets and solid surfaces, Advances in Heat Transfer 13 (1977) 1 - 60.

[3] K. Jambunathan, E. Lai, M. A. Moss, B. L. Button, A review of heat transfer data for a single circular jet impingement, International Journal of Heat Fluid Flow 13 (1992) 106 - 115.

[4] C. D. Donaldson, R. S. Snedeker, A study of free jet impingement, part i mean properties of free impinging jets, Journal of Fluid Mechanics 45 (1971) $281-319$.

[5] S. Beltaos, Oblique impingement of circular turbulent jets, Journal of Hydraulic Reserch 14 (1976) 17 - 36.

[6] R. J. Goldstein, J. F. Timmers, Visualisation of heat transfer from arrays of impinging jets, International Journal of Heat and Mass Transfer 25 (1982) $1857-1868$.

[7] R. J. Goldstein, A. I. Behbahani, K. Heppelmann, Streamwise distribution of the recovery factor and the local heat transfer coefficient to an impinging circular air jet, International Journal of Heat and Mass Transfer 29 (1986) $1227-1235$.

[8] E. M. Sparrow, B. J. Lovell, Heat transfer characteristics of an obliquely impinging circular jet, ASME Journal of Heat Transfer 102 (1980) 202 209.

[9] R. J. Goldstein, M. E. Franchett, Heat transfer from a flat surface to an oblique impinging jet, ASME Journal of Heat Transfer 110 (1988) 84 90 .

[10] X. Yan, N. Saniei, Heat transfer from an obliquely impinging circular air jet to a flat plate, International Journal of Heat and Fluid Flow 18 (1997) $591-599$.

[11] R. J. Gardon, J. C. Akfirat, The role of turbulence in determining the heat transfer characteristics of impinging jets, International Journal of Heat and Mass Transfer 8 (1965) 1261 - 1272.

[12] C. J. Hoogendoorn, The effect of turbulence on heat transfer at a stagnation point, International Journal of Heat and Mass Transfer 20 (1977) 1333 - 1338 .

[13] D. Lytle, B. W. Webb, Air jet impingement heat transfer at low nozzleplate spacings, International Journal of Heat and Mass Transfer 37 (1994) 1687 - 1697.

[14] T. Liu, J. P. Sullivan, Heat transfer and flow structures in an excited circular impinging jet, International Journal of Heat and Mass Transfer 39 (1996) $3695-3706$.

[15] S. D. Hwang, C. H. Lee, H. H. Cho, Heat transfer and flow structures in axisymmetric impinging jet controlled by vortex pairing, International Journal of Heat and Fluid Flow 22 (2001) 293 - 300.

[16] S. D. Hwang, H. H. Cho, Effects of acoustic excitation positions on heat transfer and flow in axisymmetric impinging jet: main jet excitation and 
shear layer excitation, International Journal of Heat and Fluid Flow 24 (2003) $199-209$.

[17] T. S. O'Donovan, D. B. Murray, Jet impingement heat transfer - part i: Mean and root-mean-square heat transfer and velocity distributions, International Journal of Heat and Mass Transfer 50 (2007) 3291 - 3301.

[18] T. S. O'Donovan, D. B. Murray, Jet impingement heat transfer - part ii: A temporal investigation of heat transfer and velocity distributions, International Journal of Heat and Mass Transfer 50 (2007) 3302 - 3314.

[19] J. Fitzpatrick, L. Simon, Estimation of cross-power spectra using sampleand-hold reconstruction of laser doppler anemometry data, Accepted for Publication in Experiments in Fluids.

[20] T. S. O'Donovan, Fluid flow and heat transfer of an impinging air jet, Ph.D. thesis, Department of Mechanical \& Manufacturing Engineering, Trinity College Dublin (2005).

[21] A. H. Beitelmal, M. A. Saad, C. D. Patel, The effect of inclination on the heat transfer between a flat surface and an impinging two-dimensional air jet, International Journal of Heat and Fluid Flow 21 (2000) 156 - 163.

[22] J. W. Baughn, S. S. Shimizu, Heat transfer measurements from a surface with uniform heat flux and an impinging jet, ASME Journal of Heat Transfer 111 (1989) 1096 - 1098.

[23] L. Huang, M. S. El-Genk, Heat transfer of an impinging jet on a flat surface, International Journal of Heat and Mass Transfer 37 (1994) 1915 $-1923$.

[24] P. Orlandi, R. Verzicco, Vortex rings impinging on walls: axisymmetric and three-dimensional simulations, Journal of Fluid Mechanics 256 (1993) $615-646$. 